\title{
Face Recognition Based on Symmetrical Half-Join Method using Stereo Vision Camera
}

\author{
Edy Winarno ${ }^{1}$, Agus Harjoko ${ }^{2}$, Aniati Murni Arymurthy ${ }^{3}$, Edi Winarko ${ }^{4}$ \\ ${ }^{1}$ Faculty of Information Technology, Universitas Stikubank, Semarang, Indonesia \\ ${ }^{2,4}$ Department of Computer Science and Electronics, Universitas Gadjah Mada, Yogyakarta, Indonesia \\ ${ }^{3}$ Faculty of Computer Science, Universitas Indonesia, Depok, Indonesia
}

\begin{tabular}{l}
\hline Article Info \\
\hline Article history: \\
Received Jul 14, 2016 \\
Revised Nov 06, 2016 \\
Accepted Nov 19, 2016 \\
\hline
\end{tabular}

Keyword:

Face recognition

Half-join

Stereo vision

Symmetrical

\begin{abstract}
The main problem in face recognition system based on half-face pattern is how to anticipate poses and illuminance variations to improve recognition rate. To solve this problem, we can use two lenses on stereo vision camera in face recognition system. Stereo vision camera has left and right lenses that can be used to produce a 2D image of each lens. Stereo vision camera in face recognition has capability to produce two of 2D face images with a different angle. Both angle of the face image will produce a detailed image of the face and better lighting levels on each of the left and right lenses. In this study, we proposed a face recognition technique, using 2 lens on a stereo vision camera namely symmetrical half-join. Symmetrical half-join is a method of normalizing the image of the face detection on each of the left and right lenses in stereo vision camera, then cropping and merging at each image. Tests on face recognition rate based on the variety of poses and variations in illumination shows that the symmetrical half-join method is able to provide a high accuracy of face recognition and can anticipate variations in given pose and illumination variations. The proposed model is able to produce $86 \%-97 \%$ recognition rate on a variety of poses and variations in angles between $0^{\circ}-22.5^{\circ}$. The variation of illuminance measured using a lux meter can result in $90 \%-100 \%$ recognition rate for the category of at least dim lighting levels (above 10 lux).
\end{abstract}

Copyright $@ 2016$ Institute of Advanced Engineering and Science. All rights reserved.

\section{Corresponding Author:}

Edy Winarno,

Faculty of Information Technology,

Universitas Stikubank Semarang,

Jl. Tri Lomba Juang No. 1 Semarang-50241, Jawa Tengah, Indonesia.

Email: edywin@edu.unisbank.ac.id

\section{INTRODUCTION}

Face detection is a system used to identify a human face on a static image or video from a camera [1]. Face detection system is used in several studies and further developed into multiple face tracking research to the study of face recognition [2-3]. The research field of face recognition is a research technique that combines face detection and tracking of face to know the identity of faces detected by comparing the results of the face image training that has been stored in the database and testing the facial image [4]. Generally, the face recognition system has 5-step process to identify a human face: image acquisition, face detection, normalization, feature extraction and classification [5].

In a face recognition system, the arrangement of the internal organs in the human face is used as the basis for detection and face recognition [6]. The parts of the face used as the basis for the detection of human face image are the area of the eyes, nose, mouth and cheeks. Parts of the organ on the face symmetry form a vertical pattern on the face. Pattern-based facial symmetry vertical axis would produce half a face image (half-face) of the left and right half face images that have similar parts if the mirroring process is done. Face 
recognition using half-face image would produce a facial recognition system that is relatively similar to the use of full face image (full-face), because the face has a symmetric nature [7]. Variations in the development of models of half-face image was used to anticipate the limited number and variety of models of face image during the training process [8].

The study of face recognition based on half-face image was done by [9] using the average-half-face. This research reconstructed the image of the half-face to full-face image by dividing the initial image into two equal parts between the left and right. The process of reversing the column was done on one half of the face image (left/right) and performs an average of the merger of the left and right images. The study then was continued in face recognition research by the same method, but applied in the 3D face image [10].

Face recognition research was also conducted by [8] using the pattern of half-face image in the training process. It is argued that there are four steps in the process of face recognition. The first step is to produce a face image symmetry to the training process. The face image is generated by the process of mirroring the image of the left half-face or the image of the right half-face of the frontal face position and the merger between them. The second step is the training process using the original face image to be used in the classification process. The third step is the training process of the face image symmetry to continue the process of classification. The fourth step is to use a score value of fusion of the two previous classification process to be used in face recognition.

A face recognition system that was conducted by [8] was further developed by [11] based on the pattern of half-face image. The difference between the research conducted by [11] and that conducted by [8] was the addition of the training process on the left half of the face image and the right half of the face image.

From several researches that have been done, there are some problems in a face recognition system based on a pattern of half-face image. The main problem is a variation of the face pose to produce half-face image used for face recognition accuracy. The next problem is the variation of illuminance of the face image data set in the process of face recognition accuracy [12]. Resistant to variations in face-image pose and illumination variations will affect the accuracy of face recognition results. Both of them are very important when a face recognition system will be implemented in the real-time face recognition.

The variations of poses in face recognition systems in some previous studies depended on the position of the frontal face. The accuracy of face recognition will arguably be effective if it has a face pose position near $0^{\circ}$ deviation from the frontal face. Improper position of the face in front of the camera will affect the shape and characteristics of half-face image and the image of the merger on the training process and subsequent testing, it causes a possible reduction in face recognition accuracy. These problems require solutions to anticipate the variety of poses that often occurs on the face image acquisition process. Shifting of incorrectness of the face pose of frontal face position $0^{\circ}$ at the time of the face image retrieval process can be assisted with the installation of two cameras in parallel with a certain angle in order to increase the flexibility of face poses.

Variations in lighting levels when capturing a face image will also affect to the value of accuracy on a face recognition system [12]. Lighting levels in some previous studies depends on the illumination of the front side only. This will affect the facial image and lighting detail image of a face, because the face has an uneven geometric structure. To anticipate this problems, we need a balanced lighting of the respective left and right sides of the face. The lighting in the respective left and right sides of the face can also be conducted by placing the camera on each of the left and right sides of the face. Installation of the dual cameras on the face image acquisition process will also potentially produce detailed images and maximum illumination levels on each side.

In this study, we propose a solution to anticipate variations in pose and lighting levels of face images using a stereo vision camera. Face image acquisition generates two $2 \mathrm{D}$ face images of the face detection results respectively left and right lenses in stereo vision camera. Both the $2 \mathrm{D}$ face image requires the elaboration process and produce a face image to be processed in the step of normalization, extraction and classification. This study proposes a new method to process and combine both the left and right face images into one image that has high quality information on face recognition system. The process of combining the images of left half-face and right half-face is conducted using half-join method. Half-join is a method of normalization with the cropping process on each image on the left lens and the right lens. Cropping process is conducted on the basis of the symmetric pattern of the face image (symmetrical half-join). The half of left face image is then combined with the half of right face image, which is then used as the input to the process of extraction and classification.

\section{RESEARCH METHOD}

The proposed method in this study is a model of face recognition using half-join normalization on stereo vision camera as a face image acquisition. The method employed several phases of the process of face 
recognition: face detection, normalization, feature extraction and classification. Normalization is the phase of acquisition image processing that will be used in various steps of feature extraction. Feature extraction is the process of reducing the dimension of the image. Classification is the phase to match the characteristics of image based on the characteristics of the image that has been trained. In short, the proposed process of face recognition can be seen in Figure 1.

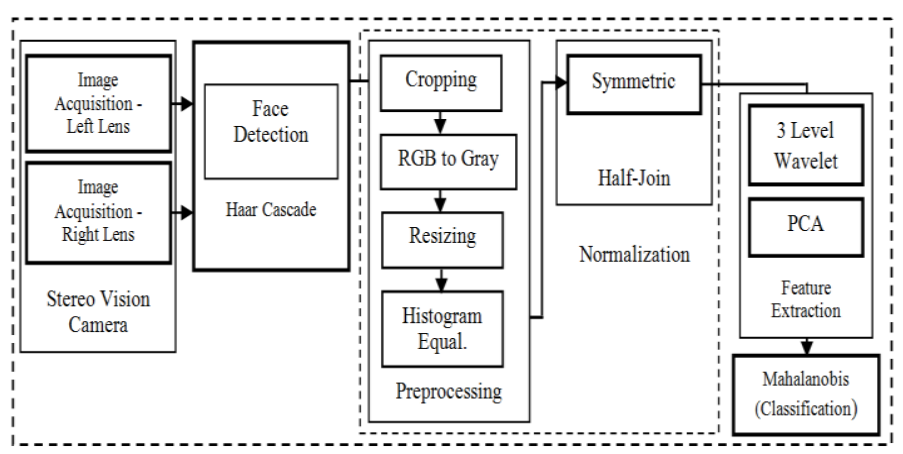

Figure 1. Proposed Process of Face Recognition

In the current study, image acquisition was conducted to capture human face images used as the information on the face detection process making use of the stereo vision camera with two lenses on the left and right. Face image was taken from frontal view with a deviation to the camera about $15^{\circ}$ on the $\mathrm{x}, \mathrm{y}$ and $\mathrm{z}$. Face detection was performed on each of the left and right lenses of a stereo vision camera. The image of the face on each lens was marked with the coordinates of the region of interest (RoI) of face image.

Face detection process was performed using the Haar Cascade Classifier [13] to determine the $\mathrm{x}$ and $y$ coordinates of the upper left corner of the image of the object, the width (w) and height (h) of image of the object. During the training process, RoI of image was normalized then stored as training data. At the testing process, RoI of the image was normalized and tested and then stored as test data.

RoI of face detection is a rectangular area in which the calculation of the coordinates of face images $\left(x_{0}, y_{0}\right),\left(x_{1}, y_{0}\right),\left(x_{0}, y_{1}\right)$ and $\left(x_{1}, y_{1}\right)$ can be used to illustrate a face image to be processed in the training and testing. Meanwhile, $\left(x_{0}, y_{0}\right)$ coordinate point is always parallel to one column with the coordinates of $\left(x_{0}, y_{1}\right)$. Coordinate point $\left(x_{0}, y_{1}\right)$ is always parallel to a line with the coordinates of $\left(x_{1}, y_{1}\right)$. Coordinate points $\left(x_{0}, y_{1}\right)$ and $\left(x_{1}, y_{1}\right)$ will vary depending on the width of the face image $(\mathrm{w})$ which detected and calculated from point $x_{0}$. Coordinate points $\left(x_{0}, y_{1}\right)$ and $\left(x_{1}, y_{1}\right)$ will also vary depending on the height image of a face (h) calculated from point $y_{0}$. Determination of RoI of face image on the face detection process is seen in Figure 2.

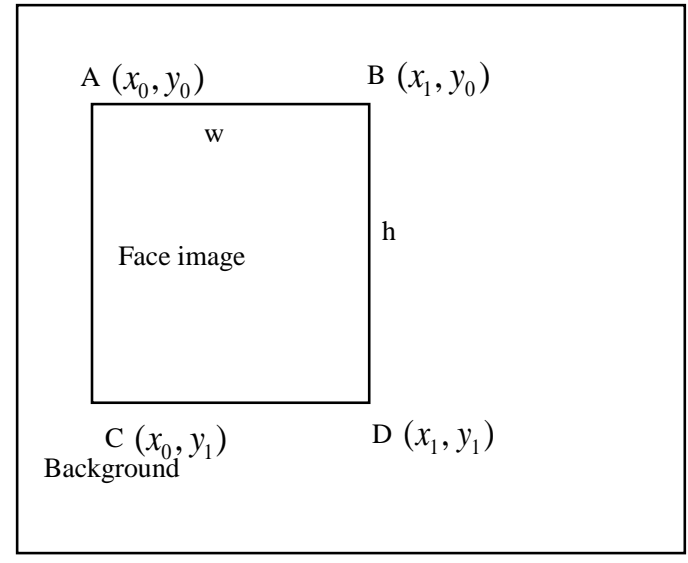

Figure 2. RoI of Face Image 
The process of calculating the RoI coordinates of face image was performed on the coordinate point $\left(x_{0}, y_{0}\right)$. The calculation was performed on considering the value of $\mathrm{w}$ and $\mathrm{h}$ of the detected face image. To determine the coordinates of all RoI of face image was obtained from the calculation of the main coordinate values $\left(x_{0}, y_{0}\right)$. The calculation to determine the point $x_{1}$ and $y_{1}$ is shown in Equations (1) and (2).

$$
\begin{aligned}
& x_{1}=x_{0}+(\mathrm{w}-1) \\
& y_{1}=y_{0}+(\mathrm{h}-1)
\end{aligned}
$$
(5) and (6).

To determine the coordinates RoI of face images A, B, C and D can be done using Equation (3), (4),

$$
\begin{aligned}
& \mathrm{A}=\left(x_{0}, y_{0}\right) \\
& \left.\mathrm{B}=\left(x_{0}+(\mathrm{w}-1)\right), y_{0}\right) \\
& \mathrm{C}=\left(x_{0}, y_{0}+(\mathrm{h}-1)\right) \\
& \mathrm{D}=\left(x_{0}+(\mathrm{w}-1), y_{0}+(\mathrm{h}-1)\right)
\end{aligned}
$$

Detected face images were then processed using normalization. The normalization process consists of two stages image processing: preprocessing stage and half-join stage. Several methods used in the preprocessing include cropping, RGB-Gray, resizing, and contrast-brightness adjustment using the histogram equalization [14]. Preprocessing methods used in this research were conducted to improve the sharpness of the image and to anticipate the variations of illuminance on face image capturing process.

The next stage was to perform a face image processing using the symmetrical half-join. Symmetrical half-join image was further processed using feature extraction and classification. We used the 3-level wavelet PCA (Principal Component Analysis) and Mahalanobis distance method as feature extraction and classification [15].

\subsection{Symmetrical Half-Join}

Half-join method is inspired by models of the human perspective using two eyes to see and identify a person. Both eyes in humans are then represented using a dual camera vision that has left and right lenses. The two images in face recognition is the application of the characteristics of the two different views simultaneously. This method combines the image of the left half-face and the right half-face into an image that is ready to be extracted. The current study used the type of half-join method namely symmetrical halfjoin.

Symmetrical half-join method cuts the image in the center of the face and produces another image of the same width. Half of the face image of the left side of the left lens was then combined with half of the face image of the right side of the right lens. The face images when combined resulted in another image with the width equal to that of its original image.

Symmetric half-join is a method of dividing the width of the face image on the left and right lenses on a camera that has the same width. The first step is to determine the width of the face image (w/ width) in pixels. The midpoint of the face image (c/center) is obtained by dividing the width of the face image into 2 parts. The image of the face of the left lens is the point of intersection of the far left $(x=0)$ up to one pixel before the midpoint of the image $(\mathrm{x}=\mathrm{c}-1)$. The image of the face of the right lens is the point of intersection of the midpoint of the image $(\mathrm{x}=\mathrm{c})$ up to the right-most point of the image $(\mathrm{x}=\mathrm{w}-1)$. The combined face image is the image of the left half of the left lens of the camera and the image of the right half of the right lens camera.

If $s h j_{\text {left }}$ is the process of cutting area of the image of the left half of the left lens of the camera, $s h j_{\text {right }}$ is the process of cutting area of the image of the right half of the right lens of the camera, $\mathrm{C}$ is the midpoint (center) and $w$ is the width resulted from detection of each image of the left and right lenses, then the value of $s h j$ is calculated and shown in equation (7)-(10). 


$$
\begin{aligned}
& s h j_{\text {left }}=\sum_{x=0}^{c-1} c \\
& s h j_{\text {right }}=\sum_{x=c}^{w-1} w \\
& \text { shj }=s h j_{\text {left }}+s h j_{\text {right }} \\
& \text { shj }=\sum_{x=0}^{c-1} c+\sum_{x=c}^{w-1} w
\end{aligned}
$$

\subsection{RoI of Half-Face Image}

Symmetric half-join has two additional RoI determining steps to produce half-face area from the left lens and half-face area from the right lens on the stereo vision camera. RoI of half-face is vertical rectangular area. RoI of left half-face and right half-face has the same width and height. The calculation of the coordinates of the top left, top right, bottom left and bottom right of half-face image is conducted in the left and right of face images on each lens on the stereo vision camera. The coordinates, width and height of the image used in the calculation are the coordinates of the RoI during face detection process. The width of halfface (c) is a half of the width of the face image (w).

\subsubsection{RoI of Left Half-Face Images}

Coordinates $\left(x_{0}, y_{0}\right)$ and $\left(x_{0}, y_{1}\right)$ are similar to the coordinates in the pixel column of RoI image in face detection. Coordinates $\left(x_{1}, y_{0}\right)$ and $\left(x_{1}, y_{1}\right)$ are also similar to the pixel column coordinates, but their positions depend on the value of the center (c) and width (w) of the face image. Coordinates $\left(x_{1}, y_{0}\right)$ and $\left(x_{1}, y_{1}\right)$ are determined depending on the midpoint (c) in the image, so the point $x_{1}$ can be calculated. Meanwhile, $y_{1}$ is determined depending on the value of width (h) of the image. RoI of left half-face image on the left lens is ilustrated in Figure 3.

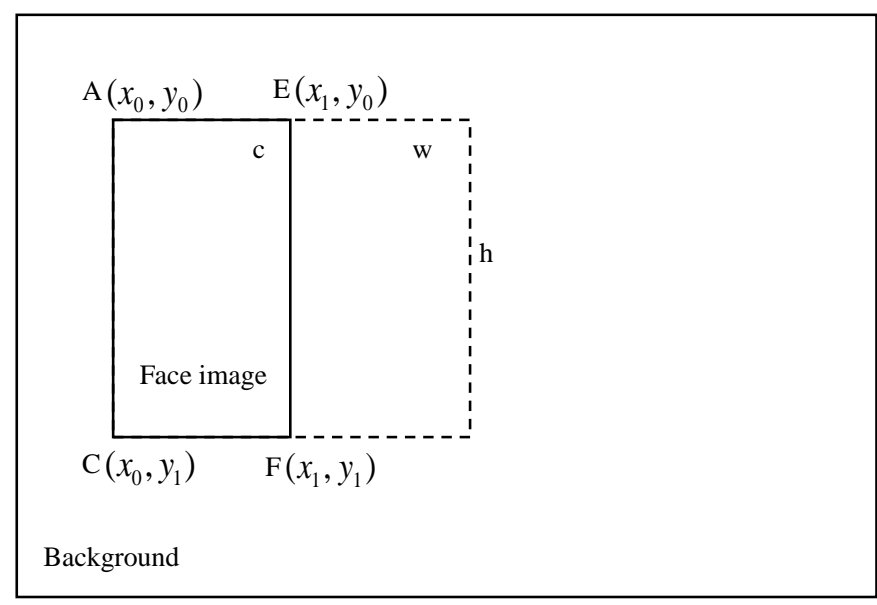

Figure 3. RoI of Left Half-Face Image on the Left-Lens

RoI of left half-face images on the left lens is calculated based on the coordinates of point A $\left(x_{0}, y_{0}\right), \mathrm{w}$ and $\mathrm{h}$. RoI of left half-face image is calculated using the midpoint of the face image (c) using Equation (11).

$$
\mathrm{c}=1 / 2 \mathrm{~W}
$$

Next, $x_{1}$ dan $y_{1}$ is calculated using Equation (12) dan (13). 


$$
\begin{aligned}
& x_{1}=x_{0}+(\mathrm{c}-1) \\
& y_{1}=y_{0}+(\mathrm{h}-1)
\end{aligned}
$$

A, E, C and F as RoI of left half-face is calculated using Equation (14)-(17).

$$
\begin{aligned}
& \mathrm{A}=\left(x_{0}, y_{0}\right) \\
& \left.\mathrm{E}=\left(x_{0}+(\mathrm{c}-1)\right), y_{0}\right) \\
& \mathrm{C}=\left(x_{0}, y_{0}+(\mathrm{h}-1)\right) \\
& \mathrm{F}=\left(x_{0}+(\mathrm{c}-1), y_{0}+(\mathrm{h}-1)\right)
\end{aligned}
$$

A, E, C and F are then combined with a right half-face on the right lens as symmetrical half-join.

\subsubsection{RoI of Right Half-Face Images}

Similar to the determination of RoI of left half face on the left lens, $\left(x_{0}, y_{0}\right)$ and $\left(x_{0}, y_{1}\right)$ in RoI of right half-face on the right lens are similar to the coordinates in the pixel column of RoI image in face detection. $\left(x_{1}, y_{0}\right)$ and $\left(x_{1}, y_{1}\right)$ are the midpoint (c) from the width of image (w). $\left(x_{2}, y_{0}\right)$ and $\left(x_{2}, y_{1}\right)$ are also similar with the pixel column coordinates, but their position depends on width (w) and height (h) from the coordinates $\left(x_{0}, y_{0}\right)$ dan $\left(x_{0}, y_{1}\right)$. RoI of right half-face image on the right lens is illustrated in Figure 4.

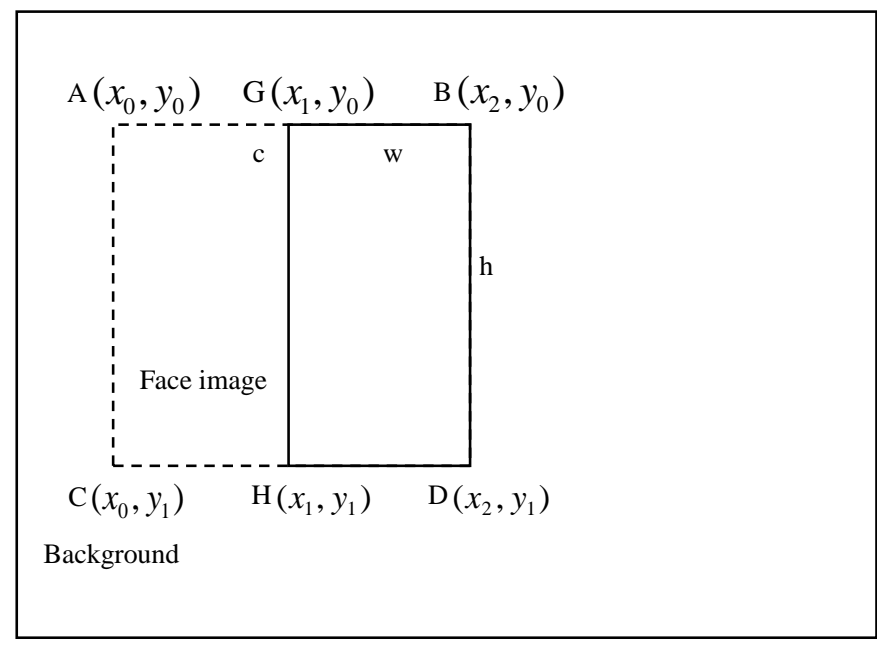

Figure 4. RoI of Right Half-Face on the Right Lens

RoI of right half-face images on the right lens was calculated based on the coordinates of point $\mathrm{A}$ $\left(x_{0}, y_{0}\right), \mathrm{w}, \mathrm{h}$ and c. Furthermore, RoI of right half-face image was calculated using the midpoint of the face image (c) using Equation (18).

$$
\mathrm{c}=1 / 2 \mathrm{~W}
$$

Then, $x_{1}, y_{1}$ and $x_{2}$ were calculated using Equation (19), (20) and (21).

$$
x_{1}=x_{0}+\mathrm{c}
$$




$$
\begin{aligned}
& y_{1}=y_{0}+(\mathrm{h}-1) \\
& x_{2}=x_{0}+(\mathrm{w}-1)
\end{aligned}
$$

G, B, H and D as RoI of right half-face were calculated using Equation (22)-(25).

$$
\begin{aligned}
& \mathrm{G}=\left(x_{0}+\mathrm{c}, y_{0}\right) \\
& \left.\mathrm{B}=\left(x_{0}+(\mathrm{w}-1)\right), y_{0}\right) \\
& \mathrm{H}=\left(x_{0}+\mathrm{c}, y_{0}+(\mathrm{h}-1)\right) \\
& \mathrm{D}=\left(x_{0}+(\mathrm{w}-1), y_{0}+(\mathrm{h}-1)\right)
\end{aligned}
$$

G, B, H and D were then combined with RoI of left half-face on the left lens (A, E, C and F) as symmetrical half-join.

\section{RESULTS AND ANALYSIS}

This study used the Viola-Jones face detection. Each left and right lenses of a stereo vision camera was used to take a raw image with the background image size of 640x480 pixels and the detected face image. Width and height of the face image depended on the distance between the face and the camera. The face image detected on the background image was marked with the coordinates of the position of the face image RoI. The face image RoI was then stored in face image storage variable. The face image was then normalized using preprocessing and half-join method.

In preprocessing, the cutting process was conducted to take the face image and it was converted into grayscale. The grayscale image was then adjusted using the resizing process to get a face image size of 160x160 pixels. The adjustment of contrast and brightness was conducted using the histogram equalization. It was used to produce the better face image quality. Image results in each of the camera lens were then processed using the method of symmetrical half-join.

The samples of results of some RoI of face image preprocessing on a stereo vision camera in some poses taken at the same time are presented in Figure 5.

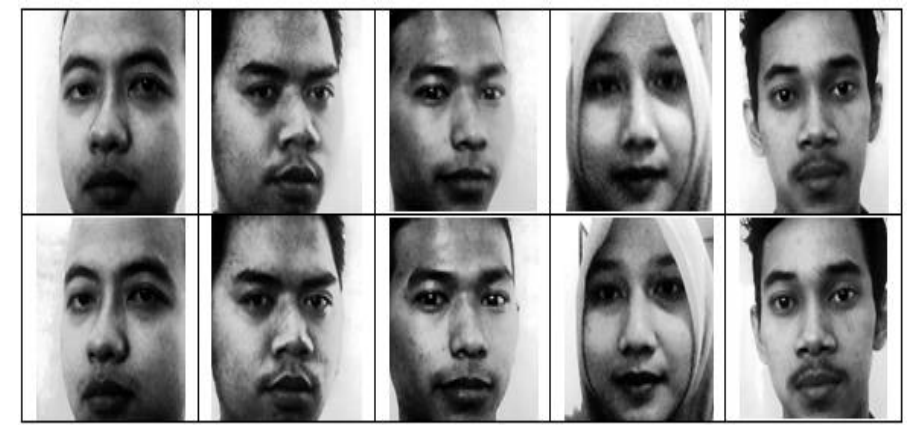

Figure 5. Face Image Preprocessing Results on a Stereo Vision Camera, (above) the Image on the Left Lens, (bottom) the Image on the Right Lens

Symmetrical half-join method cuts the image in the center of the face and produce the same image width. The size of left half-face images and right haf-face images is $80 x 160$ pixels. The left half-face image and the right half-face image are then merged into one of symmetrical half-join image. The process of merger between both of the images is illustrated in Figure 6 . 


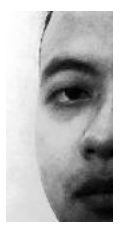

$80 \times 160$

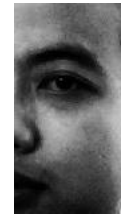

$80 \times 160$

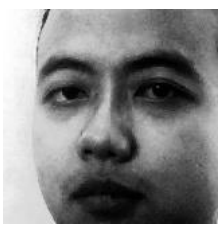

$160 \times 160$

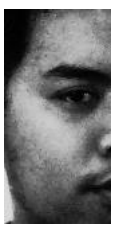

$80 \times 160$

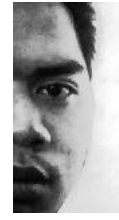

$80 \times 160$

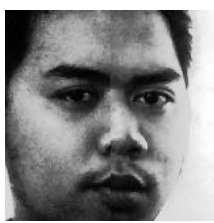

$160 \times 160$

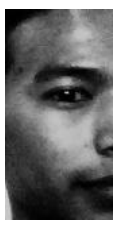

$80 \times 160$

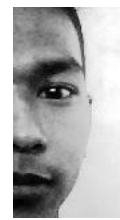

$80 \times 160$

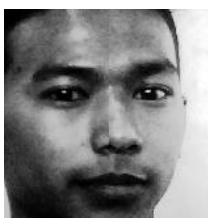

$160 \times 160$

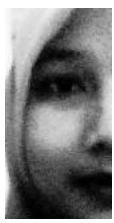

$80 \times 160$

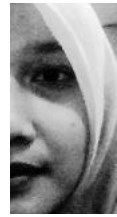

$80 \times 160$

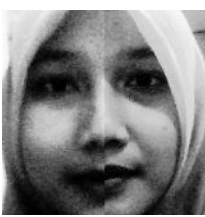

$160 \times 160$

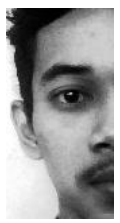

$80 \times 160$

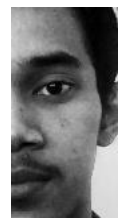

$80 \times 160$

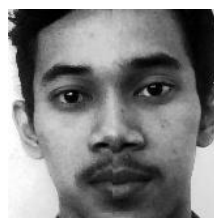

$160 \times 160$

Figure 6. (Above) The Left Half-Face of Symmetrical Half-Join, (middle) the Right Half-Face of Symmetrical Half-Join, (bottom) the Merger Image of Symmetrical Half-Join

\subsection{Face Recognition Rate on Symmetrical Half-Join}

Face recognition rate testing was conducted to compare the accuracy of the proposed model in different extraction and classification methods. We used 400 face images database of single vision and symmetrical half-join. The testing is conducted by comparing the test images that has been stored. Test image data is taken from 40 persons randomly as the test data. Test is done 4 times using random test images 100 , 200,300 and 400 of data. Test image was taken randomly from the two methods of single vision and symmetrical half-join. The comparison was conducted by comparing the face recognition rate using variations on feature extraction and classification. Summary results of face recognition rate using variations of extraction and classification are seen in Table 1.

Table 1. Summary Results of Face Recognition Rate Using Variations of Extraction and Classification

\begin{tabular}{lllll}
\hline \multirow{3}{*}{ Images } & \multicolumn{2}{l}{ Recognition rate (\%) } \\
Euclidean & Mahalanobis & \\
& PCA & 3Wavelet-PCA & PCA & 3Wavelet-PCA \\
\hline 100 & 93.00 & 92.00 & 97.00 & 94.00 \\
200 & 93.00 & 92.50 & 97.50 & 94.50 \\
300 & 94.33 & 93.00 & 97.67 & 95.33 \\
400 & 94.75 & 92.50 & 98.25 & 95.75 \\
\hline
\end{tabular}

The highest accuracy of face recognition is provided using PCA-Mahalanobis. The accuracy of face recognition using PCA-3Wavelet-Mahalanobis, PCA-Euclidean and 3Wavelet-PCA-Euclidean are over than $93 \%$.

\subsection{Recognition Rate Based on Poses Variation}

The measurement of recognition rate based on poses variation is conducted by poses of 10 persons with 7 main poses variation and 21 variations in different angles of the face image. The testing is done by 
comparing the level of accuracy of face recognition. The results of recognition rate based on face pose variation are presented in Table 2.

This research provides a level of face recognition rate on some variations of poses with different results. Generally, the testing of face recognition rate with poses variation will produce better face recognition accuracy in frontal view position, turn, bow and looked up at a maximum angle of $45^{\circ}$. The best accuracy of face recognition is in the normal position in the angle of $0^{\circ}-22.5^{\circ}$.

Table 2. Results of Recognition Rate Based on Face Pose Variation

\begin{tabular}{|c|c|c|c|c|c|c|c|}
\hline No & Pose & Angle & Recognition Rate (\%) & No & Pose & Angle & Recognition Rate (\%) \\
\hline 1 & Normal & $0^{\circ}$ & 100 & 12 & Clock wise & $67,5^{\circ}$ & 0 \\
\hline 2 & Facing left & $22,5^{\circ}$ & 97 & 13 & Anti clock wise & $22,5^{\circ}$ & 86 \\
\hline 3 & Facing left & $45^{\circ}$ & 93 & 14 & Anti clock wise & $45^{\circ}$ & 81 \\
\hline 4 & Facing left & $67,5^{\circ}$ & 34 & 15 & Anti clock wise & $67,5^{\circ}$ & 0 \\
\hline 5 & Facing left & $90^{\circ}$ & 0 & 16 & Bow & $22,5^{\circ}$ & 97 \\
\hline 6 & Facing right & $22,5^{\circ}$ & 97 & 17 & Bow & $45^{\circ}$ & 90 \\
\hline 7 & Facing right & $45^{\circ}$ & 95 & 18 & Bow & $67,5^{\circ}$ & 0 \\
\hline 8 & Facing right & $67,5^{\circ}$ & 41 & 19 & Look up & $22,5^{\circ}$ & 96 \\
\hline 9 & Facing right & $90^{\circ}$ & 0 & 20 & Look up & $45^{\circ}$ & 86 \\
\hline 10 & Clock wise & $22,5^{\circ}$ & 91 & 21 & Look up & $67,5^{\circ}$ & 34 \\
\hline 11 & Clock wise & $45^{\circ}$ & 86 & & & & \\
\hline
\end{tabular}

\subsection{Recognition Rate Based on Illuminance Variations}

Testing of face recognition rate based on variations in illumination was performed with a lux meter to measure light levels in lux (lumens $/ \mathrm{m}^{2}$ ). This tool /device was used to determine the level of variation of lighting in the room when the testing process was conducted. Testing of face recognition rate based on variations in the level of illuminance was done in $3 \times 4$ meter enclosed using variations of light as the lighting source. Testing is conducted on 10 people with 10 times taken based on the variation of illuminance. The result of face recognition rate based on illuminance variations can be seen in Table 3 .

Table 3. The Result of Face Recognition Rate Based on Illuminance Variations

\begin{tabular}{lllll}
\hline No & Iluminance (lux) & Lamps (watt) & Light Category (Based on Lux meter) & Recognition Rate (\%) \\
\hline 1 & 8 & Off & Dark $(0-10)$ & 54 \\
2 & 27 & 5 & Glimmer $(11-80)$ & 90 \\
3 & 36 & 7 & Glimmer $(11-80)$ & 91 \\
4 & 85 & 11 & Bright $(81-400)$ & 95 \\
5 & 115 & 14 & Bright $(81-400)$ & 97 \\
6 & 357 & 40 & Bright $(81-400)$ & 100 \\
7 & 482 & 60 & Very bright $(401-2500)$ & 100 \\
8 & 669 & 100 & Very bright $(401-2500)$ & 100 \\
9 & 2752 & Outdoor & Sunny $(2500<\ldots)$ & 100 \\
10 & 5361 & Outdoor & Sunny $(2500<\ldots)$ & 100 \\
\hline
\end{tabular}

Generally, the proposed model will generate the accuracy of face recognition which always stable and remains high despite the illuminance level is very low. However, to produce a high accuracy of face recognition can be performed using illuminance levels above 115 lux so that the accuracy can be maximized.

\section{CONCLUSION}

The main problem in a face recognition system based on the pattern of face symmetry is how to develop a method that can anticipate variations in facial pose and illumination variations to improve the accuracy of face recognition. In this study, we proposed a face recognition uses 2 lens on a stereo vision camera. The proposed method is an image merging from left and right face image acquisition using stereo vision camera namely half-join method. Half-join is a method of normalization of the face detection using stereo vision camera to cut on each of the images from every lens and to merge it. The merger image of the proposed method (symmetrical half-join) is then used as input to the process of extraction and classification 
in the face recognition system.

In comparison of accuracy of face recognition on each model of normalization, symmetric half-join method produces face recognition rate up to $98.25 \%$. In comparison of accuracy of face recognition based on variations in pose and illumination variations, the symmetrical half-join method is able to produce a high accuracy of face recognition and able to anticipate variations in pose and illumination. The proposed model is able to produce $86 \%-97 \%$ accuracy on a variety of poses and variations in angles between $0^{\circ}-22.5^{\circ}$. On the variation of illuminance measured using a lux meter is able to result in face recognition accuracy of $90 \%-100 \%$ for the category of lighting levels above 10 lux.

\section{REFERENCES}

[1] A.N. Martinez-Gonzalez, \& V. Ayala-Ramirez, "Real Time Face Detection Using Neural Networks", In Artificial Intelligence (MICAI), 2011 10th Mexican International Conference on, IEEE, 144-149, 2011.

[2] M.S. Devi, \& P.R. Bajaj, “Active Facial Tracking”, In Emerging Trends in Engineering and Technology (ICETET), 2010 3rd International Conference on, IEEE, 91-95, 2010.

[3] D. Sridhar \& I.M. Krishna, "Face Recognition Using Two Dimensional Discrete Cosine Transform, Linear Discriminant Analysis And K Nearest Neighbor Classifier", IAES International Journal of Artificial Intelligence, 1(4), 161, 2012.

[4] I.G.P.S. Wijaya, et al., "Face Recognition Using Holistic Features and Simplified Linear Discriminant Analysis", Indonesian Journal of Electrical Engineering and Computer Science, 10(4), 775-787, 2012.

[5] M.E. Wibowo, "Towards pose-robust face recognition on video", PhD thesis, Queensland University of Technology, 2014.

[6] W. Chen, et al., "Face detection based on half face-template", In Electronic Measurement \& Instruments, ICEMI'09, 9th International Conference on, IEEE, 4-54, 2009.

[7] P. Lekshmi, et al., "Analysis of facial expressions using PCA on half and full faces", In Audio, Language and Image Processing, 2008, ICALIP 2008, International Conference on, IEEE, 1379-1383, 2008.

[8] Y. Xu, et al., "Using the original and 'symmetrical face' training samples to perform representation based two-step face recognition, Pattern Recognition, 46, 4, 1151-1158, 2013.

[9] J. Harguess \& J.K. Aggarwal, "A case for the average-half-face in 2D and 3D for face recognition", In Computer Vision and Pattern Recognition Workshops, 2009, CVPR Workshops 2009, IEEE Computer Society Conference on, IEEE, 7-12, 2009.

[10] J. Harguess \& J.K. Aggarwal, "Is there a connection between face symmetry and face recognition?", In Computer Vision and Pattern Recognition Workshops (CVPRW), 2011 IEEE Computer Society Conference on, IEEE, 66-73 2011.

[11] M. Qiu, et al., "Integrating the original face images and "symmetrical faces" to perform face recognition", OptikInternational Journal for Light and Electron Optics, 125, 11, 2665-2670, 2014.

[12] L. Lan, et al., "Illumination Compensation for Face Recognition Using Only One Image", Acta Automatica Sinica, 39, 12, 2090-2099, 2013.

[13] P. Viola \& M.J. Jones, "Robust real-time face detection", International journal of computer vision, 57(2), 137-154, 2004.

[14] E. Winarno, et al., "Improved Real-Time Face Recognition Based On Three Level Wavelet DecompositionPrincipal Component Analysis And Mahalanobis Distance", Journal of Computer Science, 10, 5, 844-851, doi:10.3844/jcssp.2014.844.851, 2014.

[15] E. Winarno, et al., "Development Of Face Recognition System And Face Distance Estimation Using Stereo Vision Camera", Journal Of Theoretical And Applied Information Technology, 67, 3, 652-657, 2014. 